\title{
Chaotic Behavior and Strange Attractors in Dynamical Systems
}

\author{
Md. Shariful Islam Khan ${ }^{1}$ and Md. Shahidul Islam ${ }^{2}$ \\ ${ }^{1}$ Department of Mathematics, National University, Gazipur-1704, Bangladesh \\ ${ }^{2}$ Department of Mathematics, University of Dhaka, Dhaka-1000, Bangladesh
}

\begin{abstract}
$\overline{\text { Abstract : In this paper, we study the invariant set of dynamical systems in which attractor and non-attractor }}$ sets exist. We aim to carve out a small section of the theory of chaotic dynamical systems - that of attractors and outline its fundamental concepts from a computational mathematics perspective. The motivation for this paper is primarily to define what an attractor is and to clarify what distinguishes its various types (non-strange, strange non-chaotic, and strange chaotic). We discuss the Hénon and Lorenz attractors as important examples of this type of chaotic system.
\end{abstract}

Keywords: Attractor, Basin of attractor, Chaos, Strange.

\section{INTRODUCTION}

A strange attractor is basically a bounded set on which nearby orbits diverge exponentially (i.e. there exists at least one positive Lyapunov exponent). To envision such a set, consider a flat rectangle, like a piece of chewing gum. Now fold the rectangle over, stretch it, and squash it so that it maintains its original volume. Keep doing this. Two points which started out nearby to each other will eventually, after a sufficiently large number of folds and stretches, grow far apart. Formally, a strange attractor is a fractal, and may have non-integer Hausdorff dimension. (We won't discuss fractals and Hausdorff dimension here.)

Most of the examples of chaotic dynamics presented so far deal with dissipative dynamical systems, which in many instances generate chaotic attractors. Dissipative systems (i.e. the systems which lose energy by friction or diffusion) are often associated with the presence of attractors in the phase space. Roughly speaking, attractors are invariant sets to which all nearby orbits converge. They are the sets that one "sees" when a dynamical system is iterated on a computer. We have already encountered attracting fixed points and attracting periodic orbits. In practice, most of the interesting attractors are strange in the sense that either their structure is fractal or the dynamics on them is unpredictable. The interest in strange attractors increased considerably after the discovery that the phenomenon of turbulence is indeed caused by the presence of a strange attractor [1]. The term strange is most often used as a name for attractors that exhibit chaotic behaviour, that is, sensitivity to initial conditions. Though indeed true, this use of the term is somewhat misleading. It is important to clarify that strangeness is not dependent on the existence of chaos. Though attractors showing extreme sensitivity to initial conditions are indeed strange, strange attractors need not be chaotic.

\section{ATTRACTORS}

We discuss the mathematical concept of attractor, restricting ourselves to the case of topological dynamical systems $\Phi: S \times M \rightarrow M$, acting on a phase space $M$, whose topology comes from a metric $d$.

2.1. Definition: A nonempty set $\mathcal{A}$ of $M$ is said to be an attracting set for $\Phi$ if it satisfies the following two conditions:

(i) $\mathcal{A}$ is closed and invariant;

(ii) $\mathcal{A}$ possesses an open neighbourhood $U$, such that $\lim _{t \rightarrow \infty} d\left(\Phi_{t}(x), \mathcal{A}\right) \rightarrow 0$ for every $x \in U$.

The largest open set $U$ for which the condition (i) is fulfilled is called the basin of attraction of $\mathcal{A}$. The attracting sets capture the orbits which start at points in their basins of attraction.

By our definition, the union of two attracting sets is also an attracting set. We shall use the term of attractor (more precisely, open basin attractor) to designate an "irreducible" attracting set. Usually, irreducibility means topological transitivity. Alternatively, one can require that $\mathcal{A}$ has no proper attracting subsets, that is, there is no attracting set $\mathcal{A}^{\prime} \subset \mathcal{A}$ with $\emptyset \neq \mathcal{A}^{\prime} \neq \mathcal{A}$.

2.2. Theorem : Let $f: M \rightarrow M$ be a homeomorphism on a compact metric space. Then there exists a Lyapunov function $L: M \rightarrow \mathbb{R}$ such that $L$ is strictly decreasing on $M \backslash \mathcal{R}(f)$ and the image $L(\mathcal{R}(f))$ of $\mathcal{R}(f)$ is a nowhere dense set in $\mathbb{R}$. Furthermore, if $x, y \in \mathcal{R}(f)$, then $L(x)=L(y)$ if and only if $x$ and $y$ are in the same chain- 
recurrent class. That is, the chain-recurrent classes of $\mathcal{R}(f)$ are all of the form $L^{-1}(c)$, with $c$ taking values in some nowhere dense subset of $\mathbb{R}$.

Due to the Theorem 2.2, an attractor is always contained in a chain recurrent class, and conversely, any attracting set contained in a chain recurrent class is an attractor [2].

In general, an attracting set is not a collection of distinct attractors. An attractor is called global if its basin of attractor is the entire phase space. In the context of evolution equations, attractors are usually required to verify a stronger version of (ii), namely,

$$
\lim _{t \rightarrow \infty} \sup _{x \in B} d\left(\Phi_{t}(x), \mathcal{A}\right) \rightarrow 0 \text { for every bounded subset } B \subset U .
$$

2.3. Examples: (a) The following example shows that even in the one-dimensional case attracting sets can be rather complicated. Consider the flow associated with the equation

$$
\frac{d x}{d t}=-x^{4} \sin \frac{\pi}{x}
$$

It has a countable set of fixed points: 0 and $\pm 1 / n, n \in \mathbb{N}$. The interval $[-1,1]$ is an attracting set, but it contains a countable set of repelling fixed points at $\pm 1 / 2 n(n \in \mathbb{N})$ and a countable set of attracting fixed points at $\pm 1 /(2 n-1), n \in \mathbb{N}$. In fact, the linearized system at $\pm 1 / n$ is

$$
\frac{d x}{d t}=\frac{\pi}{n^{2}} \cos n x \text {. }
$$

However, the fixed point $x=0$ itself is neither a repeller nor an attracror.

(b) J. Milnor [3] introduced a more general concept of attractor for continuous maps on smooth manifolds. A Milnor attractor is a close set $\mathcal{A}$ such that (i) the realm of attraction $\rho(\mathcal{A})=\{x \in M ; \omega(x) \subset \mathcal{A}\}$ is a set of positive measure (not necessarily a neighbourhood of $\mathcal{A}$ ); (ii) there is no strictly smaller closed subset $\mathcal{A}^{\prime} \subset \mathcal{A}$ such that $\rho\left(\mathcal{A}^{\prime}\right)=\rho(\mathcal{A})$ up to a set of volume measure zero. Consider, for example, the logistic map $F_{\lambda}=$ $\lambda x(1-x)$, for $\lambda=\lambda_{\infty}=3.569 \ldots$. The limit of the period-doubling cascade has an invariant Cantor set $K \subset$ $[0,1]$, which attracts all points of $[0,1]$, except for count ably many periodic orbits of period $2^{n},(n \in \mathbb{N})[4]$. Therefore $K$ is an attractor in the sense of Milnor (known as the Feigenbaum attractor).

In practice, a way of locating attracting sets for dissipative systems is to first find a trapping region. An open subset $U_{0}$ of $M$ is called a trapping region if $\overline{\Phi_{t_{0}}\left(U_{0}\right)} \subset U_{0}$ for some $t_{0}>0$ (or, equivalently, if the vector field on the boundary of $U_{0}$ is pointing toward the interior of $U_{0}$ ). Then the existence of attracting sets is derived from the dissipative properties of the system, like the existence of bounded absorbing sets (a variant of volumecontracting). This mechanism is related to the existence of Lyapunov functions.

Let $K$ be a subset of $M$ and let $U$ be an open neighbourhood $K$. We say that $K$ is absorbing in $U$ if the orbit of every bounded subset $\mathcal{B} \subset U$ enters $K$ after a certain, i.e.,

$$
\Phi_{t}(\mathcal{B}) \subset K \text { for every } t \geq t(\mathcal{B}) \text {. }
$$

2.4. Lemma: Suppose that $M$ is a complete metric space and

$$
\Phi: S \times M \rightarrow M
$$

Is a topological dynamical system which admits a compact global absorbing set $K$. If $K$ is contained in a connected set $V$, then $K$ itself is a connected set.

Proof: If $K$ is not connected, then it can be decomposed as

$$
K=K_{1} \cup K_{2}
$$

Where $K_{1}, K_{2}$ are nonempty compact sets with $K_{1} \cap K_{2}=\emptyset$. Then $d\left(K_{1}, K_{2}\right)>0$ and for $\varepsilon>0$ small enough we have $K_{1 \varepsilon} \cap K_{2 \varepsilon}=\emptyset$, where

$$
K_{i \varepsilon}=\left\{x \in M ; d\left(x, K_{i}\right)<\varepsilon\right\}, \quad i \in\{1,2\} .
$$

But $\Phi_{t} V \subset K_{1 \varepsilon} \cup K_{2 \varepsilon}$ for $t \geq t(V)$. As $\Phi_{t} V$ is connected and $\emptyset \neq K_{i} \subset \Phi_{t} V \cap K_{i \varepsilon}$ for $i \in\{1,2\}$, we are led to a contradiction.

In what follows we shall discuss the existence of a global attractor under the presence of an absorbing set.

2.5. Theorem: Let $M$ be a complete metric space and let $\Phi: \mathrm{S} \times \mathrm{M} \rightarrow \mathrm{M}$ be a topological dynamical system which admits a compact absorbing set $K$. Then the $\omega$-limit set $\mathcal{A}=\omega(K)$ is a compact global attracting set (in fact, the maximal bounded global attracting set of $\Phi)$.

Proof: As $K$ is bounded and absorbing, there exists a $t_{0} \in S_{+}$such that

$$
\Phi_{t}(K) \subset K \text { for every } t \geq t_{0} .
$$

Put $K_{1}=\bigcup_{t \geq t_{0}} \Phi_{t}(\mathcal{A})$. Then $K_{1}$ is positively invariant and included in $K$. Consequently the closure $K^{\prime}$ of $K_{1}$ is a positively invariant compact set and thus

is compact and invariant.

$$
\mathcal{A}=\bigcap_{t \geq 0} \Phi_{t}\left(K^{\prime}\right)
$$


We shall show that $\mathcal{A}$ attracts the bounded subsets of $M$. In fact, if the contrary is true, then a bounded subset $C$ of $M$, a $\delta>0$ and a sequence $\left(t_{n}\right)_{n}$ of positive elements of $S$ would exist such that $t_{n} \rightarrow \infty$ and

$$
d\left(\Phi_{t_{n}}(C), A\right) \geq \delta \text { for every } n \in \mathbb{N} .
$$

For each $n \in \mathbb{N}$, choose a point $x_{n}$ in $C$ such that

$$
d\left(\Phi_{t_{n}}\left(x_{n}\right), A\right) \geq \delta / 2 \text {. }
$$

Because $K$ is absorbing, it follows that $\Phi_{t}(C) \subset K$ for $t$ large enough. In particular, $\Phi_{t_{n}}\left(x_{n}\right) \in K$ and $\Phi_{t}(C)$ is relatively compact for $n$ large enough. Then, passing to a subsequence if necessary, we can assume that the limit

exists. Clearly, $u \in \omega(\mathcal{A})=\mathcal{A}$, which contradicts $(1)$

$$
u=\lim _{n \rightarrow \infty} \Phi_{t_{n}}\left(x_{n}\right)
$$

As for the maximality of $\mathcal{A}$, suppose that $\mathcal{B}$ is a bounded global attracting set including $\mathcal{A}$. Then $\mathcal{B} \subset \mathcal{A}$, since $\Phi_{t}(\mathcal{B})=\mathcal{B}$ is included in $\mathcal{A}$ for $t$ large enough $(\mathcal{A}$ is absorbing in $U$ ). Consequently, $\omega(\mathcal{B})=\mathcal{B} \subset \omega(\mathcal{A})=\mathcal{A}$.

\section{HÉNON ATTRACTOR}

In 1976, the French astronomer M. Hénon [5] has initiated the study of the asymptotic dynamics of a family of smooth maps

where $a>0$ and $b$ are real parameters.

$$
H_{(a, b)}: \mathbb{R}^{2} \rightarrow \mathbb{R}^{2}, \quad H_{(a, b)}(x, y)=\left(1+y-a x^{2}, b x\right)
$$

One can show that for $(a, b)$ in a fairly large region of the parameter space, there exists a trapping region $U$ with $H_{(a, b)}(\bar{U}) \subset$ int $U$, so that $H_{(a, b)}$ has an attractive set (referred to as the Hénon attractor). Among the parameter values $(a, b)$ for which this happens are those in some small neighbourhood of $(1.4,0.3)$ and those with $1<a<2$ and $b$ close to zero.

Hénon found the values of the parameters $a=1.4, b=0.3$ after some careful explorations. If $b$ is too small, the area contraction is excessive, and the attractive set is nearly invisible. If $a$ is too small, then the attractive set consists of one attractive fixed point. Gradually increasing $a$, the attractive set successively becomes a period- 2 attractive orbit, a period- $2^{2}$ attractive orbit, a period- $2^{3}$ attractive orbit, and so on, until it eventually grows into a "one piece attractor". If $a$ is too large, almost all trajectories escape to infinity (as in the case of the logistic map $F_{\lambda}=\lambda x(1-x)$ with $\left.\lambda>4\right)$, hence there is no attractive set. Nevertheless, there are choices of parameters $a$ and $b$, with $a$ large, for which the Hénon map has an invariant set which carries chaotic dynamics [6].

Let us consider the choice of parameters $a=1.4$ and $b=0.3$, which was originally studied by Hénon. The quadrilateral $U$ of vertices $A(-1.33,0.42), B(1.32,0.133), C(1.245,-0.14), D(-1.06,-0.5)$ constitutes a trapping region for $H_{(a, b)}$, so $\mathcal{A}=\bigcap_{n \geq 0} H_{(a, b)}^{n}(U)$ is an attracting set. Since the map is dissipative (it contracts area) and invertible, the basin of attractor for the Hénon attractor (Fig. 1) must be unbounded.

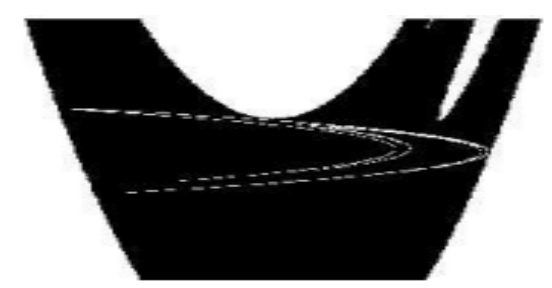

Figure 1: The basin of attraction for the Hénon attractor.

Numerical experiments suggest that the dynamics on the attractive set $\mathcal{A}$ is chaotic. They also indicate that $H_{(a, b)}$ is topologically transitive on $\mathcal{A}$, since picking a generic initial point in the plane and plotting a few thousand of iterates, its orbit always ends up in the Hénon attractor. This suggests that there is a point with dense orbit in $\mathcal{A}$, that is, $\mathcal{A}$ is an attractor (Fig. 2). Although there is no rigorous proof of these facts yet, the attractive set of Hénon is nevertheless called the Hénon attractor.

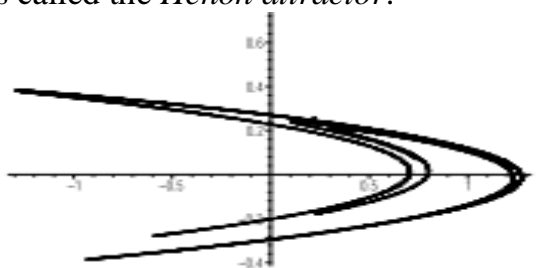

Figure 2: The Hénon attractor obtained by iterating one generic point. 
One can prove that $H_{(a, b)}$ admits a hyperbolic fixed point $p$ of coordinates

$$
x_{p}=\frac{1}{2 a}\left(-1+b+\sqrt{(1-b)^{2}+4 a}\right), y_{p}=b x_{p} ;
$$

$p$ is a saddle point since the eigenvalues of the linearized at $p$ are one negative, close to -2 , and the other positive, close to 0 . Then one can show that the closure $\overline{W^{u}(p)}$, of the unstable manifold of $p$ coincides with the Hénon attractor $\mathcal{A}$ (Fig. 3).

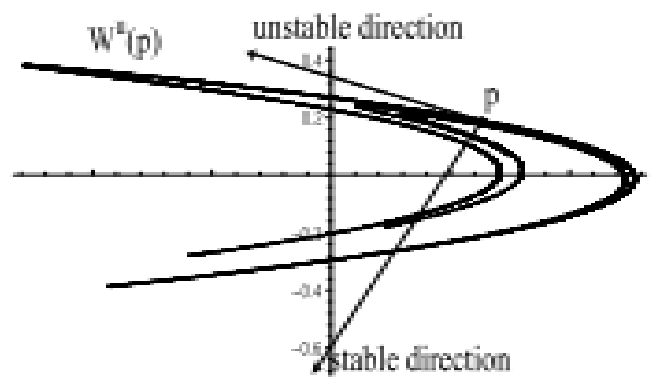

Figure 3: The stable and unstable directions at the saddle point.

The main geometric feature of the Hénon mapping is the stretching and folding of the plane. Each vertical line $\left(x_{0}, y\right)$ is mapped into a horizontal line $\left(1+y-a x_{0}^{2}, b x_{0}\right)$, while each horizontal line $\left(x, y_{0}\right)$ is mapped into a parabola $\left(1+y_{0}-a x^{2}, b x\right)$, so the quantities $a$ and $b$ control the amount of folding and stretching. Away from the $y$-axis, for example in the region $\{|x|>\sqrt{b}\}$, the dynamics is essentially hyperbolic of saddle type. One can construct an unstable cone field around the horizontal direction $(1,0)$, and a stable cone field, about the slanted direction $(1,2 a)$, which are preserved by the derivative of $H_{(a, b)}$, and, respectively, of $H_{(a, b)}^{-1}$. However these cone fields cannot be extended to the whole phase space, since horizontal segments near the $y$-axis are mapped to the turns of parabolas, due the folding that takes place in that region. For $|x|$ small, nearly horizontal vectors at $(x, y)$, inside the unstable cone, are mapped to vectors at $H_{(a, b)}(x, y)$ inside the stable cone. This phenomenon prevents $\mathcal{A}$ from having a uniformly hyperbolic structure. Nevertheless, it appears that there exists a dense set of periodic saddles in $\mathcal{A}$.

The following two theorems represent the state-of-art of the current understanding of Hénon attractor:

3.1. Theorem (M. Benedicks and L. Carleson [7]): There exista a subset $E$ (of the parameter space) with positive Lebesgue measure such that for every $(a, b) \in E$, the map $H_{(a, b)}$ admits an attractor $\mathcal{A}$ which verifies the following two properties:

(i) The basin $B(\mathcal{A})=\left\{(x, y) ; \lim _{n \rightarrow \infty} H_{(a, b)}^{n}(x, y)=\mathcal{A}\right\}$ has nonempty interior;

(ii) There exists $\left(x_{1}, y_{1}\right) \in \mathcal{A}$ whose forward orbit $O^{+}\left(x_{1}, y_{1}\right)$ is dense in $\mathcal{A}$ and there exists $c>0$ and a tangent vector $v$ to $\mathbb{R}^{2}$ at $\left(x_{1}, y_{1}\right)$ such that

for every $n \geq 1$.

$$
\left\|D H_{(a, b)}^{n}\left(x_{1}, y_{1}\right)(v)\right\|>e^{c n}\|v\|
$$

3.2. Theorem (M. Benedicks and L.-S. Young [8]): There exists a subset $E \subset \mathbb{R}^{2}$ of the parameter space with positive Lebesgue measure such that for every $(a, b) \in E$, the map $H_{(a, b)}$ admits an invariant probability measure $\mu$ such that:

(i) The support of $\mu$ is $\mathcal{A}$;

(ii) $\quad H_{(a, b)}$ has a positive Lyapunov exponent $\mu-$ a.e.;

(iii) For every continuous function $f: \mathbb{R}^{2} \rightarrow \mathbb{R}$ with compact support included in the basin of $\mathcal{A}$ and for a.e. point $(x, y)$ in the basin of $\mathcal{A}$, we have

$$
\frac{1}{n} \sum_{i=0}^{n-1} f\left(H_{(a, b)}^{i}(x, y)\right) \rightarrow \int_{\mathcal{A}} f d \mu .
$$

The measure $\mu$ in the above theorem is "physically relevant" in the sense that it describes the frequencies at which the observable orbits will visit different parts of the attractor. Its existence explains why starting with a generic point and iterating with a computer we always end up with the same picture of the Hénon attractor.

The previous two theorems, which emphasize a special kind of chaotic behavior of the dynamics on the Hénon attractor, lead to the notion of a strange attractor.

3.3. Definition (M. Viana) : An attractor $\mathcal{A}$ for a map $f$ is called a strange attractor if the following conditions are verified: 

(i) $\mathcal{A}$ contains a dense orbit along which the derivative of $f$ growth exponentially fast (in normal);
(ii) $\mathcal{A}$ contains a dense subset of periodic saddles;
(iii) $\mathcal{A}$ is the closure of the unstable manifolds of some of these saddles;
(iv) $\mathcal{A}$ supports an ergodic Sinai-Ruelle-Bowen measure $\mu$ having some positive Lyapunov exponent, and whose basin $B(\mu)$ has full Lebesgue measure within the basin of attractor of $\mathcal{A}$.
(v) $\mathcal{A}$ is persistent under small perturbation, in the sense that a generic perturbation $f_{\epsilon}$, with $f_{0}=f$, will exhibit a similar attractor for a positive Lebesgue measure set of parameters $\epsilon$.

The fact, the Hénon attractor satisfies (i), (ii) and (iii) from above, has already been explained in this section. Condition (iv) has been explained partially here (Theorem 3.2), but for more details [9]. Condition (v) is partially justified by Theorem 3.1. This type of behavior is shared by many other attractors. Moreover, these strange attractors seem to be the building blocks of any type of chaotic dynamics. It has been conjectured by J. Palis that the diffeomorphism having finitely many attractors are dense. More precisely, any diffeomorphism $f$ on a compact manifold $M$ may be approximated by another one $g$, for which there exist positively invariant open sets $U_{1}, U_{2}, \cdots, U_{k}$, with $U_{1} \cup \ldots \cup U_{k}$ of full measure in $M$, such that each point in $U_{i}$ converges under positive iteration of $g$ to either an attractive periodic orbit or to a strange attractor.

\section{LORENZ ATTRACTOR}

In 1963, the meteorologist E. N. Lorenz [10], while studying the limits of predictability of weather condition, discovered and analyzed, with the aid of a computer, a dynamical system which exhibits sensitive dependence on initial conditions. The system considered by E. N. Lorenz is

$$
\left.\begin{array}{l}
\dot{x}=\sigma(y-x) \\
\dot{y}=r x-y-x z \\
\dot{z}=x y-b z
\end{array}\right\}
$$

where $\sigma, r, b$ are parameters.

This system is a simplified model of the convective motion of a two dimensional fluid cell warmed from above and cooled from below, as in Fig. 4 . In brief, $x$ measures the rate of convective overturning, $y$ measures the horizontal overturning, and $z$ measures the vertical overturning of the fluid across the cell. The parameter $\sigma$ is proportional to the Prandtl number, which represents the ratio of the kinematic viscosity to its thermal conductivity, the parameter $r$ is proportional to the Rayleigh number, which represents the difference in temperature between the top and bottom of the system, and the parameter $b$ is proportional to the physical proportions of the cell. All three parameters are thus positive since they represent physical quantities.

The most commonly studied values of the parameters are $\sigma=10, r=28, b=8 / 3$. It is however important to observe the structural changes in the dynamics for fixed $\sigma=10$ and $b=b / 3$, while increasing $r$ from 0 to $\infty$.

The divergence of the flow

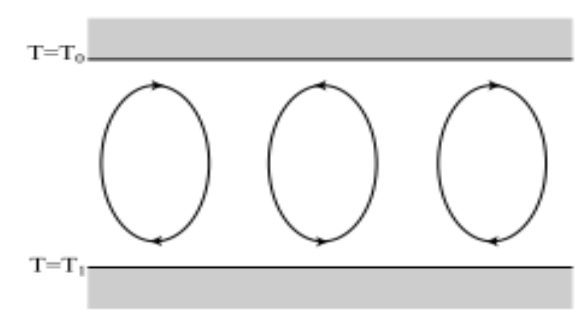

Figure 4: Lorenz cells.

$$
\nabla \cdot(\dot{x}, \dot{y}, \dot{z})=\partial \dot{x} / \partial x+\partial \dot{y} / \partial y+\partial \dot{z} / \partial z=-(\sigma+b+1)
$$

is negative, so the volume element $d(\mathrm{Vol})$ is contracted by the flow to $d(\Phi(t) \mathrm{Vol})=e^{-(\sigma+b+1)} d(\mathrm{Vol})$ in a time interval of $t$. This shows that the system is dissipative, which is a strange indication for the existence of some attractive set. In order to find a trapping region, we define a Lyapunov function

Its derivative along solution curves is

$$
V(x, y, z)=r x^{2}+\sigma y^{2}+\sigma(z-2 r)^{2} .
$$

$$
\frac{d V}{d t}=-2 \sigma\left(r x^{2}+y^{2}+b z^{2}-2 b r z\right)
$$

There is a bounded region $D$, given by the equation

$$
r x^{2}+y^{2}+b z^{2} \leq 2 b r z
$$

on which $d V / d t \geq 0$. Let $c$ be the maximum value of $V$ on this region and let $U$ be an ellipsoid defined by $V<c+\varepsilon$, containing $D$, for some small $\varepsilon$. So we have $d V / d t<-\delta$, for some $\delta>0$ depending on $\varepsilon$, and for all points outside $U$. For the forward orbit $\Phi_{t}(x)$ of any point outside the ellipsoid, the Lyapunov function $V\left(\Phi_{t}(x)\right)$ will decrease at a rate bounded away from zero. This says that all trajectories starting outside of $U$ 
will eventually meet the surface of the ellipsoid, then immediately enter its interior, and then remain there for ever. In other words, $U$ is a trapping region, and moreover, a global absorbing set.

Then Theorem 2.5 implies the existence of a global attractive set for the trajectories of the system 4 . Considering a sequence of instants $t=1<2<\cdots$ approaching infinity, we notice that the flow successively takes the ellipsoid $U$ into the regions $\Phi_{1}(U), \Phi_{2}(U), \ldots$, for which we have $\operatorname{Vol}\left(\Phi_{n}(U)\right)=e^{-(\sigma+b+1) n} \operatorname{Vol}(U)$. Since on the surface of the ellipsoid $U$ the flow lines point inwards, we have $\Phi_{1}(\bar{U}) \subset U$, thus

$$
U \supset \Phi_{1}(U) \supset \Phi_{2}(U) \supset \cdots \supset \Phi_{n}(U) \cdots
$$

Hence the attractor $\mathcal{A}=\bigcap_{n \geq 0} \Phi_{n}(U)$ has zero volume.

It is clear that the origin is a fixed of the flow. If $0<r \leq 1$, one can easily check that it is the only fixed point. We now show that for $0<r<1$ the origin is a globally asymptotically stable equilibrium point. To this end, we consider a different Lyapunov function

Its derivative along solution curves is

$$
W(x, y, z)=x^{2}+\sigma y^{2}+\sigma z^{2} .
$$

$$
\frac{d W}{d t}=2 \sigma\left((1+r) x y-x^{2}-y^{2}-b z^{2}\right) .
$$

By completing the square, it follows that $d W / d t<0$ for all points $(x, y, z) \neq(0,0,0)$. Thus the desired conclusion is achieved. The same conclusion can be achieved by using characteristic exponents, as below. The case of $0<r<1$ corresponds physically to the case of no motion and steady heat conduction across the cell.

If $r>1$, then there exist 3 fixed points, the origin and the points $p_{1}, p_{2}$ of coordinates

$$
x=y= \pm \sqrt{b(r-1)}, \quad z=r-1
$$

The stability of these points can be analyzed using characteristic exponents. The system (2) linearized at the origin becomes

and its eigenvalues are

$$
\left.\begin{array}{l}
\dot{x}=\sigma(y-x) \\
\dot{y}=r x-y \\
\dot{z}=-b z
\end{array}\right\}
$$

$$
\lambda_{1}, \lambda_{2}=\frac{1}{2}\left[-\sigma-1 \pm\left((\sigma-1)^{2}+4 r \sigma\right)^{1 / 2}\right], \lambda_{3}=-b .
$$

For $r<1$ all eigenvalues are negative, so the origin is stable; for $r>1, \lambda_{1}$ is positive and $\lambda_{2}, \lambda_{3}$ are negative, so the origin is unstable. Thus the origin is a hyperbolic fixed point of saddle type, with the unstable manifold 1dimensional, and the stable manifold 2-dimensional. The stable manifold contains the $z$-axis, looks flat near the origin and it cannot intersect the trajectories starting on the unstable manifold of the origin, but other than that it is hard to describe. The system (2) linearized at the fixed points $p_{1}, p_{2}$ is

and its characteristic polynomial is

$$
\left.\begin{array}{l}
\dot{x}=\sigma(y-x) \\
\dot{y}=r x-y \mp b \sqrt{r-1} \\
\dot{z}= \pm b \sqrt{r-1} x \pm b \sqrt{r-1}-b z
\end{array}\right\}
$$

$$
P(\lambda)=\lambda^{3}+(\sigma+b+1) \sigma^{2}+b(\sigma+r) \lambda+2 \sigma b(r-1) .
$$

The roots of this polynomial may be all real or one real and two complex, depending on the value of $r$. In any case, there exists a special value

$$
r_{h}=\sigma(\sigma+b+3) /(\sigma-b-1) \approx 24.74
$$

of $r$ (usually referred as a critical $r$-value), such that for all $1<r<r_{h}$, all of the eigenvalues of the linearized system at $p_{1}$ and $p_{2}$ have negative real part. Accordingly, these two fixed points are asymptotically stable. The corresponding maximal attractor consists of $p_{1}, p_{2}$ and the unstable manifold of the origin, which gets arbitrarily near the fixed points $p_{1}$ and $p_{2}$ ( Fig. 5).

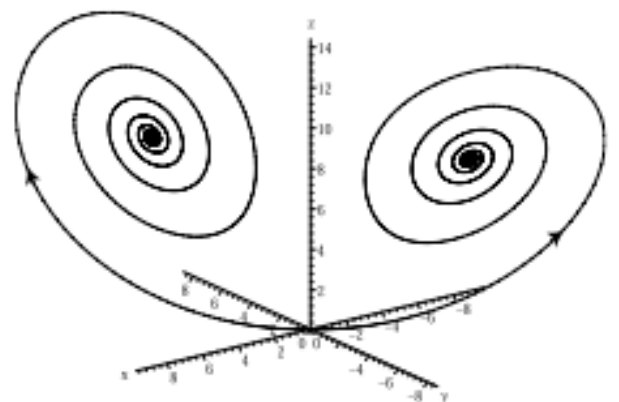

Figure 5: The unstable manifold of the origin. 
There exists an intermediate value $r_{0} \in\left(1, r_{h}\right), r_{0} \approx 1.346$, such that for $1<r<r_{0}$, the characteristic exponents of $p_{1}$ and $p_{2}$ are negative, while for $r_{0}<r<r_{h}$, two of them are conjugate complex numbers, with a negative real part, in which case the unstable manifold of the origin circles around $p_{1}$ and $p_{2}$. There exists another intermediate value $p_{1} \approx 13.296$ for which the unstable manifold of the origin connects to the stable manifold of the origin, forming a homoclinic loop. Thus, trajectories starting from the unstable manifold of the origin will eventually land in the stable manifold of the origin, and will then tend, in both forward and backward time, to the origin (Fig. 6). The case of $1<r<r_{h}$ corresponds physically to a motion of the fluid governed by steady convection rolls in either of two senses.

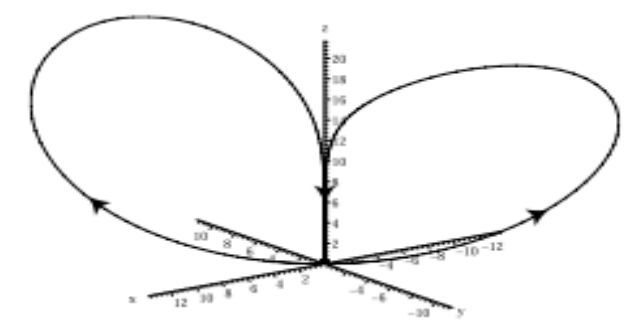

Figure 6: A homoclinic orbit.

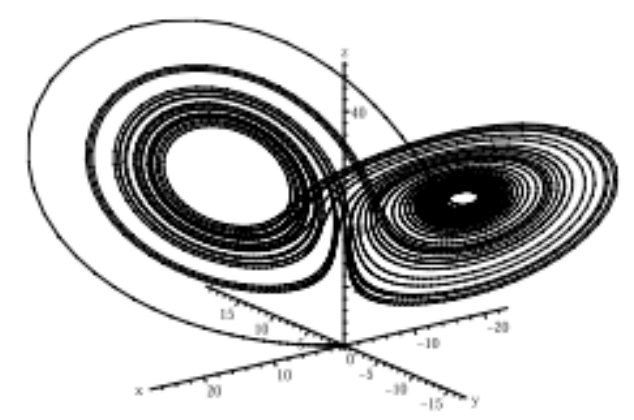

Figure 7: A trajectory starting nearby the origin and trapped by the Lorenz attractor.

For $r>r_{h}$ all the three fixed points are unstable and a chaotic attractor is observable. The attractor lies on an infinitely ramified surface, with trajectories running from one branch to another in a chaotic fashion (Fig. 7). This situation corresponds physically to the destruction of the steady convection rolls by a turbulent, large amplitude motion. If we increase $r>28$, the chaotic features of the Lorenz attractor seems to persist for a wide range of $r$. For very large values of $r(r>313)$, the trajectories tend to become relatively simpler and attractive periodic orbits appear.

\section{CONCLUSION}

The primary focus of this paper is to gain an understanding of what it means to be an attractor and how this idea relates to chaos. In particular, we explore the meaning of strangeness. It is a common misconception that the term strange attractor is simply another way of saying chaotic attractor. Though a chaotic attractor is certainly a strange attractor, the reverse is not necessarily true. Indeed, there exist examples of strange attractors which are non-chaotic.

\section{References}

[1] Ruelle D. and Takens F., On the nature of turbulence, Commun. Math. Phy., 20 (1971), 167-192, and 23 (1971), $343-344$.

[2] Temam R., Infinite dimensional dynamical systems in Mechanics and Physics, Springer Verlag, 1988.

[3] Milnor J., On the concept of attractor, Commun. Math. Phy., 99 (1985), 177-195.

[4] Guckenheimer J., Sensitive dependence to initial conditions for one dimensional maps, Commun. Math. Phy., 70 (1979), 133-160.

[5] Hénon M., A two dimensional mapping with a strange attractor, Commun. Math. Phy., 50 (1976), 69-77.

[6] Khan M. S. I. and Islam M. S., Hyperbolic Dynamics in Two Dimensional Maps, Int. J. Pure Appl. Sci. Technol., 11(1) (2012), 5766.

[7] Benedicks M. and Carleson L., The dynamics of Hénon map, Annals of Mathematics, 133 (1991), 73-169.

[8] Benedicks M. and Young L.-S., Sinai-Bowen-Ruelle measures for certain Hénon maps, Inventions Math., 112 (1993), 541-576.

[9] Gidea, Marian and Niculescu, Constantin P., Chaotic dynamical systems: An introduction, Craiova University Press, 2002.

[10] Lorenz, E. N., Deterministic nonperiodic flows, Journal of the atmospheric sciences, 20 (1963), 130-141. 